\title{
Bacteria and genes associated with arsenite oxidation and nitrate reduction in a paddy soil
}

XIAOMIN LI $^{1 *}$, SHUANG $\mathrm{LI}^{3}$, JiANGTAO QIAO ${ }^{2}$, FANGBAI $\mathrm{LI}^{2}$

${ }^{1}$ SCNU Environmental Research Institute, Guangdong Provincial Key Laboratory of Chemical Pollution and Environmental Safety, South China Normal University, Guangzhou 510006, China (*correspondence: xiaomin.li@m.scnu.edu.cn)

${ }^{2}$ Guangdong Key Laboratory of Integrated Agroenvironmental Pollution Control and Management, Guangdong Institute of Eco-Environmental Science \& Technology, Guangzhou 510650, China

${ }^{3}$ Guangdong Bioengineering Institute (Guangzhou Sugarcane Industry Research Institute), Guangdong Key Laboratory of Sugarcane Improvement and Biorefinery, Guangzhou 510316, China

Recent studies demonstrated that microbial arsenite (As(III)) oxidation associated with nitrate $\left(\mathrm{NO}_{3}^{-}\right)$reduction might be an important process in diminishing arsenic bioavailability and toxicity to rice when paddy soils are contaminated by arsenic. However, the responses of bacterial communities and functional genes to As(III) under nitratereducing conditions are poorly understood in a noncontaminated soil. In this study, our results revealed that microbial oxidation of $\mathrm{As}(\mathrm{III})$ to $\mathrm{As}(\mathrm{V})$ was substantially accelerated by nitrate addition in a paddy soil with low arsenic background content, while nitrate reduction was not affected by As(III) addition. Metagenomic binning revealed that Pseudogulbenkiania sp. was the main nitrate-reducing bacteria with narG, nirS and norBC genes, and Azoarcus sp. was the predominant As(III)-oxidizing bacteria with As(III) oxidase genes aioA and aioB. The draft genome of Azoarcus sp. also had complete sets of denitrification genes (mainly, napA, nirK, and nosZ). Quantitive PCR analysis also confirmed that the abundance of Azoarcus spp., aioA and nosZ genes were enhanced in the presence of As(III) and nitrate. These findings suggest the importance of Azoarcusand Pseudogulbenkiania-related spp., both of which showed various physio-ecological characteristics for arsenic and nitrogen biogeochemistry, in coupling As(III) oxidation and nitrate reduction in flooded paddy soil.

This work was supported by National Natural Science Foundation of China (41877043 and 41907130) and Guangdong Natural Science Funds for Distinguished Young Scholars (2017A030306010). 